\section{A retrospective analysis of two-month sputum smear non-conversion in new sputum smear positive tuberculosis patients in the Free State Province, South Africa}

\author{
Nanteza Gladys Kigozi, 1 \\ Perpetual Chikobvu, ${ }^{2,3}$ \\ James Christoffel Heunis, ${ }^{1}$ \\ Sonja van der Merwe ${ }^{3}$ \\ ${ }^{1}$ Centre for Health Systems Research \& \\ Development, University of the Free \\ State; ${ }^{2}$ Department of Community \\ Health, University of the Free State; \\ ${ }^{3}$ Free State Department of Health, \\ Bloemfontein, South Africa
}

\section{Abstract}

Little is known about the drivers of twomonth sputum smear non-conversion in the South African context. Our study sought to determine these factors in new sputum smear positive tuberculosis (TB) patients in South Africa's Free State Province. A retrospective record review was conducted for all TB patients on treatment between 2003 and 2009. Twomonth sputum smear non-conversion was defined by a positive sputum smear result. Data was subjected to univariate, bivariate and regression analyses. Generalized linear regression models were used to estimate the risk for two-month sputum smear non-conversion. Age, pre-treatment sputum smear grading, HIV status and TB disease classification influenced two-month sputum smear non-conversion. Significant associations were thus established between health systems, microbiological, clinical and demographic factors, and two-month sputum smear non-conversion. This study provides program managers with evidence to support the development of more tailored TB care.

\section{Introduction}

Despite the availability of highly effective treatment, tuberculosis (TB) remains a serious problem in South Africa. The World Health Organization (WHO) assessment showed that TB incidence in all the 22-high burden countries is falling except in South Africa. The country in fact accounted for one-quarter of all incident TB cases in the WHO Africa Region in 2010. ${ }^{1}$ Two-month sputum smear conversion is a useful indicator of TB program performance.
Research has shown that persistent sputum positivity at the end of two months of TB treatment can predict adverse treatment outcomes including increased failure and/or relapse rates. $^{2-4}$ In terms of TB patient management, two-month sputum smear conversion is an important measure of treatment progress. From our preliminary investigations of the routine data for $2009,83 \%$ of the TB patients in the Free State Province of South Africa who were classified as cured at the end of treatment had also had successful sputum smear conversion during the intensive (first two months) treatment phase. ${ }^{5}$ The results of sputum smear testing at the end of the intensive phase determine whether the anti-TB regimen can be switched to the continuation phase or whether the patient should be given an additional month of intensive phase treatment. ${ }^{6-8}$ Knowledge of the factors associated with persistent sputum positivity at the end of two months of anti-TB therapy is therefore useful for clinicians to better manage their patients and improve treatment outcomes.

Previous studies have associated sputum conversion/non-conversion with age ${ }^{2-4,9,10}$ pretreatment sputum smear grading [acid fast bacilli (AFB) $]^{3,4,11-15}$ and sex., ${ }^{2,10,14,16}$ To our knowledge, no studies have examined the issue of sputum non-conversion in the Free State Province. This is of concern since the incidence of new smear positive TB has been steadily increasing countrywide since $2002 .{ }^{17}$ The current research set out to establish the factors associated with two-month sputum smear non-conversion in the Free State. Based on literature, it was hypothesized that twomonth sputum smear non-conversion would be associated with delayed treatment onset after positive sputum smear diagnosis, pre-treatment sputum smear grading, HIV status, TB disease classification, and patient demographics including age and sex.

\section{Materials and Methods}

\section{Study design and setting}

A retrospective record review was conducted on routine data in the ETR.Net (http://www.etrnet.info/WhatIsETR.aspx) for all new smear positive TB patients in the Free State who received treatment between 2003 and 2009. The ETR.Net is software designated for the capturing of TB patient information directly from standardized paper registers. At facility level, TB patients' clinical information is collated onto standardized paper TB registers by the TB nurse. The information on the paper registers is then validated by the local area TB coordinator. A copy of the validated information is then sent to be captured on the ETR.Net
Correspondence: Gladys Kigozi, University of the Free State, P.0. Box 339, (Internal Box 39), Bloemfontein, 9300 South Africa.

Tel.: +27.51.401.3333 - Fax: +27. 51.401.9694

E-mail: kigozign@ufs.ac.za

Key words: tuberculosis, sputum smear non-conversion, Free State Province, South Africa.

Contributions: NGK, initial draft writing and manuscript revision; PC, data analysis and comments on the manuscript; JCH, study design and comments towards manuscript revision; SvdM, study conceptualization, project management and comments towards manuscript revision.

Conflict of interests: NGK and JCH declare no financial interest or potential conflict of interest; $\mathrm{PC}$ is the acting director of strategic information management at the Free State Department of Health; SvdM is a Free State Department of Health manager responsible for technical and clinical support.

Acknowledgements: the Free State Department of Health is acknowledged for supporting this research.

Received for publication: 24 January 2013.

Revision received: 12 February 2014.

Accepted for publication: 19 February 2014.

This work is licensed under a Creative Commons Attribution NonCommercial 3.0 License (CC BYNC 3.0).

(C) Copyright N.G. Kigozi et al., 2014

Licensee PAGEPress, Italy

Journal of Public Health in Africa 2014; 5:324

doi:10.4081/jphia.2014.324

at the sub-district level. Once data is captured in the ETR.Net, it is available on a server and can be accessed at both the district and provincial levels. Data for the present study was aggregated for all health care facilities in the five districts comprising the Free State Province.

\section{Study population}

The study population was defined as new smear positive TB patients eight years and older registered in the ETR. Net during the stated period. Excluded from the study were patients who were transferred out, those who had died or had interrupted treatment before the end of the intensive phase and those whose sputum results were not recorded (including those who could not produce sputum) at the end of two months on treatment.

\section{Measures}

Sputa are collected according the South African national TB treatment guidelines; at the end of the initial two months of treatment 
and again at treatment completion. Two sputum specimens are collected for smear examination at each point in time. ${ }^{6}$ The current study concentrated on sputum smear results at two months of TB treatment. The outcome variable, two-month sputum smear non-conversion, was measured by a positive sputum smear result - confirmed by presence of TB bacilli - recorded at the end of the intensive phase of treatment.

Independent variables considered for the study included: age in years $(8-17,18-27,28$ $37,38-47,48-57$ or $\geq 58$ ); pre-treatment sputum smear grading (scanty, AFB $1^{+}, \mathrm{AFB} 2^{+}, \mathrm{AFB} 3^{+}$, not recorded); number of days from date of TB diagnosis to date of treatment onset (0-4, 5-7, $8-11$ or $\geq 12$ ); HIV status (positive, negative, not recorded); TB disease classification [pulmonary tuberculosis (PTB) or both PTB and extra-pulmonary tuberculosis (EPTB)] and sex (male or female). All clinical data were confirmed by routine laboratory tests recorded in the relevant paper registers and the ETR.Net.

We used the age category of 8-17 years in order to determine non-conversion rates among children whose compliance is presumably monitored by their parents. The subsequent categories, i.e. 18-27, 28-37, 38-47, 4857 , and $\geq 58$ years were arbitrary increments in age and were used to determine the effect of increasing age on two-month sputum smear non-conversion. The pre-treatment sputum smear grading was based on results of the flu- orescence microscopy with auramine 0 stain method, routinely recorded in the TB register. HIV status was based on routinely recorded rapid antibody tests. ${ }^{18}$ The number of days from TB diagnosis to treatment was determined by the difference in days between date of treatment onset and the date of TB diagnosis. While sub-groups for this variable were arbitrarily determined, practice in the province is that patients should be initiated on treatment within five days of TB diagnosis. The first category was therefore $0-4$ days. TB disease classification was based on the national protocol for TB diagnosis. ${ }^{6}$

\section{Analysis}

Data was subjected to univariate, bivariate and regression analyses using Stata version 10 (StataCorp, College Station, TX, USA). We used generalized linear models to estimate the risk for two-month sputum smear non-conversion for each sub-group of independent variables, adjusting for other independent variables in the model. The variables considered for both univariate and multivariate models include age, pre-treatment sputum grading, treatment onset delay, HIV status and TB disease classification. Risk ratios (RR) together with their corresponding $95 \%$ confidence intervals (CIs) were estimated for the regression models. Two-sided significance was considered at $P \leq 0.05$. Due to statistically signifi- cant differences between males and females regarding the outcome variable, results are presented for each of the sexes as well as the population as a whole (Tables 1 and 2).

\section{Ethics approval}

The study was approved by the Health Research Ethics Committee at the University of Stellenbosch and the Ethics Advisory Board of the International Union Against Tuberculosis and Lung Disease.

\section{Results}

\section{Retrospective record review}

A total of 54,164 records were extracted from the ETR.Net. Of these, 5256 (9.7\%) cases were transferred out, 2903 (5.4\%) were reported dead, 877 (1.6\%) had interrupted treatment before the end of the intensive phase, and sputum smear results were not recorded at the end of two months on treatment for 5141 (9.5\%) cases (those who could not produce sputum included). A total of 39,987 (73.8\%) cases had a recorded two-month sputum smear result.

\section{Patient demographics}

The total number of cases with a recorded two-month sputum result included 21,444

Table 1. Distribution of two-month sputum smear results across sex groups.

\begin{tabular}{|c|c|c|c|}
\hline Variable & $\begin{array}{c}\text { Male }(n=21,444) \\
\text { Number }(\%)\end{array}$ & $\begin{array}{c}\text { Female }(\mathrm{n}=\mathbf{1 8 , 5 4 3 )} \\
\text { Number }(\%)\end{array}$ & $\begin{array}{c}\text { Total }(\mathrm{n}=39,987) \\
\text { Number }(\%)\end{array}$ \\
\hline $\begin{array}{l}\text { Age (years)* } \\
08-17 \\
18-27 \\
28-37 \\
38-47 \\
48-57 \\
58 \geq\end{array}$ & $\begin{array}{c}750(3.5) \\
3806(17.8) \\
6943(32.4) \\
5885(27.4) \\
2974(13.9) \\
1086(5.1)\end{array}$ & $\begin{array}{c}1140(6.2) \\
5311(28.6) \\
6190(33.4) \\
3673(19.8) \\
1490(8.0) \\
739(4.0)\end{array}$ & $\begin{array}{c}1890(4.7) \\
9117(22.8) \\
13,133(32.8) \\
9558(23.9) \\
4464(11.2) \\
1825(4.6)\end{array}$ \\
\hline $\begin{array}{l}\text { Pre-treatment sputum } \\
\text { Scanty } \\
\text { AFB 1+ } \\
\text { AFB 2+ } \\
\text { AFB 3+ } \\
\text { Not recorded }\end{array}$ & $\begin{array}{l}\text { ding } \\
1844(8.6) \\
4666(21.8) \\
3080(14.4) \\
7721(36.0) \\
4133(19.3)\end{array}$ & $\begin{array}{l}2016(10.8) \\
4300(23.2) \\
2870(15.5) \\
5966(32.2) \\
3391(18.3)\end{array}$ & $\begin{array}{c}3860(9.7) \\
8966(22.4) \\
5950(14.9) \\
13,687(34.2) \\
7524(18.8) \\
\end{array}$ \\
\hline $\begin{array}{l}\text { Delay from diagnosis t } \\
\quad 0-4 \\
5-7 \\
8-11 \\
\geq 12\end{array}$ & $\begin{array}{r}\text { nt onset (days) } \\
6609(30.8) \\
6077(28.3) \\
3840(17.9) \\
4918(22.9)\end{array}$ & $\begin{array}{l}5340(28.8) \\
4933(26.6) \\
3453(18.6) \\
4817(26.0)\end{array}$ & $\begin{array}{c}11,949(30.0) \\
11,010(27.5) \\
7293(18.2) \\
9735(24.4)\end{array}$ \\
\hline $\begin{array}{l}\text { HIV status } \\
\text { Positive } \\
\text { Negative } \\
\text { Not recorded }\end{array}$ & $\begin{array}{c}2934(13.7) \\
1397(6.5) \\
17,113(79.8) \\
\end{array}$ & $\begin{array}{c}3242(17.5) \\
920(5.0) \\
14,381(77.6)\end{array}$ & $\begin{array}{c}6176(15.4) \\
2317(5.8) \\
31,494(78.8) \\
\end{array}$ \\
\hline Disease classification & & & \\
\hline $\begin{array}{l}\text { Both PTB and EPTB } \\
\text { PTB only }\end{array}$ & $\begin{array}{c}537(2.5) \\
20,907(97.5) \\
\end{array}$ & $\begin{array}{c}468(2.5) \\
18,075(97.5) \\
\end{array}$ & $\begin{array}{c}1005(2.5) \\
38,982(97.5) \\
\end{array}$ \\
\hline
\end{tabular}

\footnotetext{
*Mean age ( \pm standard deviation): males=37.4 ( \pm 11.9$)$ years; females=33.6 ( \pm 12.1$)$ years; total=35.4 ( \pm 12.1$)$ years. AFB, acid fast bacilli; PTB, pulmonary tuberculosis; EPTB, extra-pulmonary tuberculosis
} 
(53.6\%) males and 18,543 (46.4\%) females (Table 1). Over the study period, respectively $12.5 \%$ of male, $9.3 \%$ of female and $11.0 \%$ of all cases registered sputum smear non-conversion at two months of TB treatment. The mean ( \pm standard deviation) age was $37.4( \pm 11.9)$ years for males, $33.6( \pm 12.1)$ years for females and $35.4( \pm 12.1)$ years for the whole population. A substantial proportion of the population (32.8\%) were aged between 28 and 37 (males: $32.4 \%$; females: $33.4 \%$ ) years. The pre-treatment sputum smear grading was very severe $\left(\mathrm{AFB} 3^{+}\right.$) in approximately one-third (34.2\%) of the total population. Severe pre-treatment grading was $36.0 \%$ in males and $32.2 \%$ in females. Treatment onset had been delayed by 12 days or more following TB diagnosis in about one-quarter (24.4\%) of the cases in the population. Substantial proportions of both male (22.9\%) and female (26.0\%) cases had delayed treatment onset of 12 days and more. HIV test results were available for only 8493 of the TB cases in the entire population. Just over three-quarters (77.9\%) of females and twothirds $(67.7 \%)$ of males were co-infected. It should be noted that the large proportion (78.8\%) of unrecorded HIV test results (males: 79.8\%; females: $77.6 \%$ ) resulted from the fact that capturing of HIV status as part of routine TB data commenced only in 2006. In addition, routine (opt-out) HIV testing for TB patients was only effected in 2007, following the endorsement of the Tuberculosis Strategic Plan for South Africa, 2007-2011. ${ }^{19}$

\section{Factors associated with two-month sputum smear non-conversion}

All variables considered were independently statistically significantly $(\mathrm{P}<0.001)$ associated with sputum smear non-conversion at two months of TB treatment. At $12.5 \%$, males cases had a significantly $(\mathrm{P}<0.001)$ higher rate of two-month sputum smear non-conversion than females at $9.3 \%$. Among the age groups, those aged between 48-57 years reported a significantly $(\mathrm{P}<0.001)$ higher rate of non-conversion relative to other age groups. As expected, non-conversion was significantly highest among cases with very severe (AFB $3+$ ) pretreatment grading $(18.5 \% ; \quad \mathrm{P}<0.001)$. Surprisingly, patients who delayed treatment initiation by more than 12 days had statistically significantly lower non-conversion (9.8\%; $\mathrm{P}<0.001)$ than to those who had delayed treatment onset by 11 days or less. HIV-negative cases registered statistically significantly $(\mathrm{P}<0.001)$ higher non-conversion $(12.0 \%)$ than HIV-positive cases (8.4\%) and those with unrecorded HIV status (11.5\%). Non-conversion was also significantly higher in cases diagnosed with PTB only (11.1\%) than those classified to have both PTB and EPTB (7.06\%; $\mathrm{P}<0.001$ ) (Table 3).

\section{Risk for two-month sputum smear} non-conversion

In Table 2, results indicate that after controlling for other variables in the model, male patients aged between 48 and 57 years were at $140 \%$ greater risk for two-month sputum smear non-conversion compared to their counterparts aged 8-17 years (adjusted RR, 2.4; CI, 1.8-3.3). Among female cases, those aged 58 and older were at $30 \%$ greater risk of non-conversion compared to those aged 8-17 years old (adjusted RR, 1.3; CI, 1.01-1.75). In the population as a whole, patients aged between 48 and 57 years faced significantly higher risk of non-conversion than those aged 8-17 years (adjusted RR, 1.8; CI, 1.5-2.1).

Regarding the pre-treatment sputum grading, male cases with a sputum smear grading of $\mathrm{AFB} 3^{+}$were at a $480 \%$ higher risk (adjusted RR, 5.8; CI, 4.6-7.5) for two-month smear non-conversion compared to their counterparts with a scanty grading. Likewise, females with a sputum smear grading of AFB $3^{+}$had a $430 \%$ higher risk for two-month sputum smear non-conversion relative to those with a scanty grading (RR, 5.3; CI, 4.1-6.8). A similar trend was observed for cases with AFB $3^{+}$ grading in the general population who had a $470 \%$ higher risk of non-conversion than those with scanty grading (adjusted RR, 5.7; CI, 4.8-6.8).

Table 2. Factors associated with two-month sputum smear non-conversion among new smear positive male and female PTB patients

\begin{tabular}{|c|c|c|c|c|c|c|c|c|c|}
\hline \multirow[t]{2}{*}{ Variable } & \multicolumn{3}{|c|}{ Male $(n=2686)$} & \multicolumn{3}{|c|}{ Female $(n=1727)$} & \multicolumn{3}{|c|}{ Total ( $\mathrm{n}=4413)$} \\
\hline & $\begin{array}{c}\% \text { NC } \\
\text { RR (CI) }\end{array}$ & $\begin{array}{l}\text { Univariate } \\
\text { RR (CI) }\end{array}$ & $\begin{array}{l}\text { Adjusted } \\
\text { (CI) }\end{array}$ & $\begin{array}{l}\% \text { NC } \\
\text { RR (CI) }\end{array}$ & $\begin{array}{l}\text { Univariate } \\
\text { (CI) }\end{array}$ & $\begin{array}{l}\text { Adjusted } \\
\text { RR }\end{array}$ & $\% \mathrm{NC}$ & $\begin{array}{c}\text { Univariate RR } \\
\text { (CI) }\end{array}$ & $\begin{array}{l}\text { Adjusted } \\
\text { RR }\end{array}$ \\
\hline $\begin{array}{l}\text { Age (years) } \\
\text { 08-17 (ref) } \\
18-27 \\
28-37 \\
38-47 \\
48-57 \\
58 \geq\end{array}$ & $\begin{array}{c}1.6 \\
17.1 \\
30.8 \\
28.6 \\
16.5 \\
5.4\end{array}$ & $\begin{array}{c}1.0 \\
2.1(1.5-2.8)^{*} \\
2.0(1.5-2.7)^{*} \\
2.2(1.7-3.0)^{*} \\
2.5(1.9-3.4)^{*} \\
2.3(1.7-3.2)^{*}\end{array}$ & $\begin{array}{c}1.0 \\
1.7(1.3-2.3)^{*} \\
1.9(1.4-2.5)^{*} \\
2.1(1.6-2.8)^{*} \\
2.4(1.8-3.3)^{*} \\
2.3(1.7-3.2)^{*}\end{array}$ & $\begin{array}{c}5.9 \\
29.2 \\
31.9 \\
20.4 \\
8.1 \\
4.5\end{array}$ & $\begin{array}{l}1.0 \\
1.1(0.9-1.3) \\
1.0(0.8-1.2) \\
1.1(0.9-1.3) \\
1.1(0.8-1.3) \\
1.2(0.9-1.5)\end{array}$ & $\begin{array}{c}1.0 \\
1.1(0.9-1.3) \\
1.1(0.9-1.4) \\
1.2(1.01-1.53)^{* *} \\
1.2(1.0-1.6) \\
1.3(1.01-1.75)^{* *}\end{array}$ & $\begin{array}{l}3.31 \\
21.8 \\
31.2 \\
25.4 \\
13.2 \\
5.1\end{array}$ & $\begin{array}{c}1.0 \\
1.4(1.2-1.6)^{*} \\
1.4(1.2-1.6)^{*} \\
1.5(1.3-1.8)^{*} \\
1.7(1.4-2.0)^{*} \\
1.6(1.3-1.9)^{*}\end{array}$ & $\begin{array}{c}1.0 \\
1.3(1.1-1.5)^{* *} \\
1.4(1.2-1.6)^{*} \\
1.6(1.4-1.9)^{*} \\
1.8(1.5-2.1)^{*} \\
1.7(1.4-2.1)^{*}\end{array}$ \\
\hline $\begin{array}{l}\text { Pre-treatment sputum smear } \\
\text { Scanty (ref) } \\
\text { AFB 1+ } \\
\text { AFB 2+ } \\
\text { AFB 3+ } \\
\text { Unrecorded }\end{array}$ & $\begin{array}{r}\text { rrading } \\
2.4 \\
10.2 \\
11.8 \\
58.3 \\
17.3\end{array}$ & $\begin{array}{c}1.0 \\
1.7(1.3-2.2)^{*} \\
3.0(2.3-3.9)^{*} \\
5.8(4.6-7.5)^{*} \\
3.2(2.5-4.2)^{*}\end{array}$ & $\begin{array}{c}1.00 \\
1.7(1.3-2.2)^{*} \\
3.0(2.3-3.8)^{*} \\
5.8(4.5-7.4)^{*} \\
3.2(2.5-4.1)^{*}\end{array}$ & $\begin{array}{c}3.6 \\
11.4 \\
11.4 \\
56.1 \\
17.7\end{array}$ & $\begin{array}{c}1.0 \\
1.5(1.1-2.0)^{* *} \\
2.2(1.7-2.9)^{*} \\
5.3(4.1-7.0)^{*} \\
2.9(2.2-3.8)^{*}\end{array}$ & $\begin{array}{c}1.0 \\
1.5(1.1-2.0)^{* *} \\
2.2(1.7-3.0)^{*} \\
5.3(4.1-6.8)^{*} \\
2.8(2.1-3.6)^{*}\end{array}$ & $\begin{array}{c}2.9 \\
10.6 \\
11.7 \\
57.4 \\
17.4\end{array}$ & $\begin{array}{c}1.0 \\
1.6(1.3-2.0)^{*} \\
2.6(2.2-3.2)^{*} \\
5.7(4.8-6.8)^{*} \\
3.1(2.6-3.8)^{*}\end{array}$ & $\begin{array}{c}1.0 \\
1.6(1.3-1.9)^{*} \\
2.6(2.2-3.2)^{*} \\
5.7(4.8-6.8)^{*} \\
3.0(2.5-3.7)^{*}\end{array}$ \\
\hline $\begin{array}{l}\text { Delay from diagnosis to treat } \\
0-4 \text { (ref) } \\
5-7 \\
8-11 \\
\geq 12\end{array}$ & $\begin{array}{c}\text { ent onset } \\
29.0 \\
30.3 \\
20.0 \\
20.7\end{array}$ & $\begin{array}{l}\text { (days) } \\
1.0 \\
1.1(1.04-1.25)^{* *} \\
1.2(1.07-1.31)^{* *} \\
1.0(0.9-1.1)\end{array}$ & $\begin{array}{c}1.0 \\
1.1(1.0-1.2) \\
1.2(1.04-1.27)^{* *} \\
1.0(0.9-1.1)\end{array}$ & $\begin{array}{l}29.9 \\
27.4 \\
19.4 \\
23.3\end{array}$ & $\begin{array}{c}1.0 \\
1.00(0.9-1.1) \\
1.00(0.9-1.1) \\
0.9(0.76-0.98)^{* *}\end{array}$ & $\begin{array}{c}1.0 \\
0.9(0.8-1.0) \\
1.0(0.9-2.0) \\
0.9(0.8-1.0)\end{array}$ & $\begin{array}{l}29.4 \\
29.2 \\
19.8 \\
21.7\end{array}$ & $\begin{array}{c}1.0 \\
1.08(1.0-1.2) \\
1.1(1.02-1.20)^{* *} \\
0.9(0.84-0.98)^{* *}\end{array}$ & $\begin{array}{c}1.0 \\
1.0(0.9-1.1) \\
1.0(1.0-1.2) \\
1.0(0.9-1.0)\end{array}$ \\
\hline $\begin{array}{l}\text { HIV status } \\
\text { Positive (ref) } \\
\text { Negative } \\
\text { Not recorded }\end{array}$ & $\begin{array}{c}11.2 \\
7.6 \\
81.2 \\
\end{array}$ & $\begin{array}{c}1.0 \\
1.4(1.2-1.7)^{*} \\
1.2(1.1-1.4)^{*}\end{array}$ & $\begin{array}{c}1.0 \\
1.2(1.01-1.40)^{* *} \\
1.2(1.06-1.33)^{* *}\end{array}$ & $\begin{array}{c}12.7 \\
4.2 \\
83.0\end{array}$ & $\begin{array}{c}1.0 \\
1.2(0.9-1.5) \\
1.5(1.3-1.7)^{*}\end{array}$ & $\begin{array}{c}1.0 \\
1.0(0.8-1.3) \\
1.5(1.3-1.7)^{*}\end{array}$ & $\begin{array}{c}11.8 \\
6.3 \\
81.9 \\
\end{array}$ & $\begin{array}{c}1.0 \\
1.4(1.2-1.6)^{*} \\
1.4(1.2-1.5)^{*}\end{array}$ & $\begin{array}{c}1.0 \\
1.2(1.04-1.36)^{* *} \\
1.3(1.2-1.4)^{*}\end{array}$ \\
\hline $\begin{array}{l}\text { Disease classification } \\
\text { Both PTB and EPTB (ref) } \\
\text { PTB only }\end{array}$ & $\begin{array}{c}1.5 \\
98.5\end{array}$ & $\begin{array}{c}1.0 \\
1.7(1.3-2.3)^{* *}\end{array}$ & $\begin{array}{c}1.0 \\
1.4(1.06-1.91)^{* *}\end{array}$ & $\begin{array}{c}1.8 \\
98.2\end{array}$ & $\begin{array}{c}1.0 \\
1.4(1.0-2.0)\end{array}$ & $\begin{array}{c}1.0 \\
1.3(0.9-1.8)\end{array}$ & $\begin{array}{l}1.61 \\
98.4\end{array}$ & $\begin{array}{c}1.0 \\
1.6(1.3-2.0) * 1\end{array}$ & $\begin{array}{c}1.0 \\
1.4(1.09-1.70)^{* *}\end{array}$ \\
\hline
\end{tabular}


Male cases with delayed treatment initiation of 8-11 days were at a $20 \%$ greater (adjusted RR, 1.2; CI, 1.04-1.27) risk for two-month sputum smear non-conversion compared to their male counterparts with less than five days delay in treatment onset. These differences were however not statistically significant after controlling for other variables in the model within the female sub group, as well as the entire population.

Relative to their HIV-positive counterparts, HIV-negative males (adjusted RR, 1.2; CI, 1.01.4) and those with unknown HIV status (adjusted RR, 1.2; CI, 1.1-1.3) both had 20\% higher risk for non-conversion. Among female cases, those with unrecorded HIV test results had a $50 \%$ higher risk for two-month sputum smear non-conversion compared to their HIVpositive counterparts (RR, 1.5; CI, 1.3-1.7). In the general population, HIV-negative cases and those with unrecorded status had respectively $20 \%$ (RR, 1.2; CI, 1.04-1.36) and 30\% (RR, 1.3; $\mathrm{CI}, 1.2-1.4)$ greater risk of non-conversion than their HIV-positive counterparts.

Regarding TB disease classification, after controlling for other variables, results were statistically significant only among male cases and in the general population. Males diagnosed with only PTB had 40\% higher risk for non-conversion compared to males diagnosed with both PTB and EPTB (RR, 1.4; CI, 1.061.91). In the general population, cases diagnosed with only PTB also had 40\% higher risk for sputum smear non-conversion relative to those with both PTB and EPTB (RR, 1.4; CI, 1.09-1.70).

\section{Discussion}

Results showed an association between age and two-month sputum smear non-conversion, with older TB patients showing high risk for non-conversion. These findings are consistent with studies conducted among TB patients in Saudi Arabia ${ }^{3}$ and Burkina Faso. ${ }^{10}$ Progressive age-related immune dysregulation and delayed TB diagnosis ${ }^{20,21}$ could possibly account for the observed two-month sputum smear non-conversion among older patients in the current study. These poor smear conversion rates emphasise the need for more rigorous clinical management of older PTB patients.

In line with research conducted in other settings, ${ }^{3,4,11-15}$ patients with severe pre-treatment sputum smear grading had a higher risk for two-month sputum smear non-conversion than those with scanty grading. As shown in a Saudi Arabian study, ${ }^{3}$ patients with a high pretreatment sputum smear grading not only showed poor sputum conversion at the end of the intensive treatment phase, but were also more likely to exhibit poor treatment outcomes including lower cure rate, and higher failure and death rates, than patients with a scanty grading. Hence, to avoid such poor outcomes, TB programmes need to intensify active TB case finding.

The finding that male patients who had delayed treatment initiation by $8-11$ days had a $20 \%$ higher risk for two-month sputum smear non-conversion compared to their male counterparts with less than five days delay cannot be readily explained from the current study and should be further investigated.

In this study, HIV-negative TB patients were at a higher risk for two-month sputum smear non-conversion than their HIV-positive counterparts. It could be that the specialist-driven treatment adherence training received by HIVpositive patients also had a positive effect on these patients when they initiated their TB treatment, consequently influencing their sputum conversion. However, previous research in Tanzania $^{22}$ and Uganda ${ }^{23}$ has shown that HIV status is not a significant predictor of twomonth sputum smear non-conversion.

Patients diagnosed with only PTB were at a higher risk for two-month sputum smear nonconversion than their counterparts diagnosed with both PTB and EPTB. This finding could possibly be attributed to the fact that patients diagnosed with both PTB and EPTB are usual- ly hospitalized and receive specialist management, with directly observed treatment by nurses. Consequently, compliance to treatment is high with reduced risk for non-conversion.

Limitations of the study include: firstly, some patients did not have sputum smear results because the process of grading, capturing and monitoring of sputum smear results as part of routine data commenced only during the period 2004-2005; secondly, there is potential misclassification of the outcome variable as culture investigations were not considered; thirdly, it was not possible to establish the reasons for delay in commencing TB treatment after diagnosis; fourthly, findings relating to HIV status should be interpreted with caution as the HIV results were not recorded for most of the patients due to the aforementioned reasons; and fifthly, the study was based only on the routinely collected data and therefore did not consider other important variables that could influence the outcome variable, including chest radiograph results, patient symptoms at diagnosis, as well as nutritional status. Nonetheless, as far as could be established, this is the first study in South Africa which has assessed the determinants of two-month sputum smear non-conversion using routinely recorded patient information.

Our findings have two important policy

Table 3. Association between certain variables and two-month sputum smear outcome

\begin{tabular}{|c|c|c|c|}
\hline \multicolumn{3}{|c|}{ Variable $\quad$ Two-month sputum smear outcome $(n=39,987)$} & P-value \\
\hline $\begin{array}{l}\text { Gender } \\
\quad \text { Male }(\mathrm{n}=21,444) \\
\text { Female }(\mathrm{n}=18,543)\end{array}$ & $\begin{array}{l}18,758(87.5) \\
16,816(91.0)\end{array}$ & $\begin{array}{c}2686(12.5) \\
1727(9.3)\end{array}$ & $<0.001$ \\
\hline $\begin{array}{c}\text { Age (years) } \\
08-17(\mathrm{n}=1890) \\
18-27(\mathrm{n}=9477) \\
28-37(\mathrm{n}=13,133) \\
38-47(\mathrm{n}=9658) \\
48-57(\mathrm{n}=4464) \\
58 \geq(\mathrm{n}=1825)\end{array}$ & $\begin{array}{c}1744(92.3) \\
8513(89.4) \\
11,758(89.5) \\
8436(88.3) \\
3881(86.9) \\
1602(87.8)\end{array}$ & $\begin{array}{l}146(7.7) \\
964(10.6) \\
1375(10.5) \\
1122(11.7) \\
583(13.1) \\
223(12.2)\end{array}$ & $<0.001$ \\
\hline $\begin{array}{l}\text { Pre-treatment sputum sme } \\
\text { Scanty }(\mathrm{n}=3860) \\
\text { AFB } 1+(\mathrm{n}=8966) \\
\text { AFB } 2+(\mathrm{n}=5950) \\
\text { AFB } 3+(\mathrm{n}=13,687) \\
\text { Not recorded }(\mathrm{n}=7524)\end{array}$ & $\begin{array}{l}\text { ar grading } \\
3734(96.7) \\
8497(94.8) \\
5435(91.3) \\
11,153(81.5) \\
6755(89.8)\end{array}$ & $\begin{array}{c}126(3.3) \\
469(5.2) \\
515(8.7) \\
2534(18.5) \\
769(10.2)\end{array}$ & $<0.001$ \\
\hline $\begin{array}{l}\text { Delay from diagnosis to tre } \\
\quad 0-4(\mathrm{n}=11,949) \\
5-7(\mathrm{n}=11,010) \\
8-11(\mathrm{n}=7293) \\
\geq 12(\mathrm{n}=9735)\end{array}$ & $\begin{array}{c}\text { atment onset }( \\
10,653(89.1) \\
9722(88.3) \\
6421(88.0) \\
8778(90.2)\end{array}$ & $\begin{array}{c}1296(10.9) \\
1288(11.7) \\
872(12.0) \\
957(9.8)\end{array}$ & $<0.001$ \\
\hline $\begin{array}{l}\text { HIV status } \\
\text { Positive }(\mathrm{n}=6176) \\
\text { Negative }(\mathrm{n}=2317) \\
\text { Not recorded }(\mathrm{n}=31,494\end{array}$ & $\begin{array}{c}5655(91.6) \\
2039(88.0) \\
27,880(88.5)\end{array}$ & $\begin{array}{c}521(8.4) \\
278(12.0) \\
3614(11.5)\end{array}$ & $<0.001$ \\
\hline $\begin{array}{l}\text { Disease classification } \\
\text { Both PTB and EPTB } \\
\text { PTB only }\end{array}$ & $\begin{array}{c}934(92.9) \\
34,640(88.9)\end{array}$ & $\begin{array}{c}71(7.1) \\
4342(11.1)\end{array}$ & $<0.001$ \\
\hline
\end{tabular}

AFB, acid fast bacilli; PTB, pulmonary tuberculosis; EPTB, extra-pulmonary tuberculosis. 
implications for TB program performance and resource allocation in the Free State: Firstly, TB patients' characteristics including age, sex, pre-treatment sputum smear grading, time to treatment initiation, HIV status and TB disease classification need to be considered when monitoring TB program outcomes. Secondly, efforts need to be made towards early TB patient identification to avoid high levels of pre-treatment sputum smear grading and subsequent treatment failure/relapse.

\section{Conclusions}

Findings support our hypothesis that twomonth sputum smear non-conversion is associated with patient demographics, including age and sex, as well as delayed treatment onset, pre-treatment sputum smear grading, HIV status, and TB disease classification. Significant associations were thus established between health systems, microbiological, clinical and demographic factors and two-month sputum smear non-conversion. The study provides program managers with evidence to support the development of more tailored care.

\section{References}

1. World Health Organization (WHO). Global tuberculosis control. WHO Report 2011. Geneva: WHO; 2011.

2. Kuaban C, Barne R, Mouanue L, et al. Nonconversion of sputum smears in new smear positive pulmonary tuberculosis patients in Yaoundé, Cameroon. East Afr Med J 2009;86:219-25.

3. Singla R, Osman MM, Khan N, et al. Factors predicting persistent sputum smear positivity among pulmonary tuberculosis patients 2 months after treatment. Int J Tuberc Lung Dis 2003;7:58-64.

4. Banu Rekha VV, Balasubramanian R, Swaminathan S, et al. Sputum conversion at the end of intensive phase of Category-
1 regimen in the treatment of pulmonary tuberculosis patients with diabetes mellitus or HIV infection: an analysis of risk factors. Indian J Med Res 2007;126:452-8.

5. Free State Department of Health (FSDoH). ETR.NET database. Bloemfontein: FSDoH; 2012.

6. Department of Health (South Africa $\mathrm{NDoH}$ ). National tuberculosis management guidelines. Pretoria: NDoH; 2009.

7. Enarson D, Rieder H, Arnadottir T, Trébucq A. Management of tuberculosis: a guide for low-income countries. 5th ed. Paris: International Union Against Tuberculosis and Lung Disease; 2000.

8. Trébucq A, Rieder HL. Two excellent tools for national tuberculosis programmes: history of previous treatment and sputum status at two months. Int $\mathrm{J}$ Tuberc Lung Dis 1998;2:184-6.

9. Domínguez-Castellano A, Muniain MA, Rodriguez-Baño J, et al. Factors associated with time to sputum smear conversion in active pulmonary tuberculosis. Int $\mathrm{J}$ Tuberc Lung Dis 2003;7:432-8.

10. Dembele SM, Ouedraogo HZ, Combary A. Conversion rate at two month follow-up of smear positive tuberculosis patients in Burkina Faso. Int $\mathrm{J}$ Tuberc Lung Dis 2007;11:1339-44.

11. Singla R, Singla N, Sarin R, Arora VK. Influence of pre-treatment bacillary load on treatment outcome of pulmonary tuberculosis patients receiving DOTS under revised National Tuberculosis Control Programme. Indian J Chest Dis Allied Sci 2005;47:19-23.

12. Gopi PG, Chandrasekaran V, Subramani R, et al. Association of conversion and cure with initial smear grading among new smear positive pulmonary tuberculosis patients treated with Category I regimen, Ind J Med Res 2006;123:807-14.

13. Fortún J, Martín-Dávila P, Molina A, et al. Sputum conversion among patients with pulmonary tuberculosis: are there implications for removal of respiratory isolation? J Antimicrob Chemother 2007;59:794-8.

14. Jayawardena KAS, Samarathunga M. Baseline characteristics of patients and the effect of fixed-dose combination chemotherapy on sputum conversion time in active pulmonary tuberculosis: a preliminary study in Kandy District, Sri Lanka. SAARC J Tuberc Lung Dis HIV/AIDS 2008;5:1-6.

15. Wang J, Fei Y, Shen H, Xu B. Gender difference in knowledge of tuberculosis and associated health-care seeking behaviors: a cross-sectional study in a rural area of China. BMC Public Health 2008;8:354.

16. Güler M, Unsal E, Dursun B, et al. Factors influencing sputum smear and culture conversion time among patients with new case pulmonary tuberculosis. Int $\mathrm{J}$ Clin Pract 2007;61:231-5.

17. Day C, Gray A. Health and related indicators. In: Fonn S, Padarath, eds. South African health review 2010. Durban: Health Systems Trust; 2010. pp 211-364.

18. Department of Health (South Africa $\mathrm{NDoH})$. National HIV counselling and testing (HCT) policy guidelines. Pretoria: NDoH; 2010.

19. Department of Health (South Africa $\mathrm{NDoH})$. Tuberculosis strategic plan for South Africa, 2007-2011. Pretoria: NDoH; 2007.

20. Gaur SN, Dhingra VK, Rajpal S, et al. Tuberculosis in the elderly and their treatment outcome under DOTS. Indian $\mathrm{J}$ Tuberc 2004;51:83-7.

21. Davies PD0. The effects of poverty and ageing on the increase in tuberculosis. Monaldi Arch Chest Dis 1999;54:168-71.

22. Senkoro M, Mfinanga SG, Mørkve 0 . Smear microscopy and culture conversion rates among smear positive pulmonary tuberculosis patients by HIV status in Dar es Salaam, Tanzania. BMC Inf Dis 2010;210:10.

23. Bwire R, Borgdorff MW, Sticht-Groh V, et al. Tuberculosis chemotherapy and sputum conversion among HIV sero-positive and HIV sero-negative patients in southeastern Uganda. East Afr Med J 1999;76:3 07-13. 